\title{
The exploration of meditation in the neuroscience of attention and consciousness
}

\author{
Antonino Raffone $\cdot$ Narayanan Srinivasan
}

Received: 4 December 2009/Accepted: 11 December 2009/Published online: 30 December 2009

(C) Marta Olivetti Belardinelli and Springer-Verlag 2009

\begin{abstract}
Many recent behavioral and neuroscientific studies have revealed the importance of investigating meditation states and traits to achieve an increased understanding of cognitive and affective neuroplasticity, attention and self-awareness, as well as for their increasingly recognized clinical relevance. The investigation of states and traits related to meditation has especially pronounced implications for the neuroscience of attention, consciousness, self-awareness, empathy and theory of mind. In this article we present the main features of meditation-based mental training and characterize the current scientific approach to meditation states and traits with special reference to attention and consciousness, in light of the articles contributed to this issue.
\end{abstract}

Keywords Meditation - Consciousness - Attention · Self-awareness · Neuroplasticity

\section{Introduction}

Meditation can be conceptualized as a family of complex emotional and attentional regulatory practices, in which

A. Raffone $(\square)$

Department of Psychology, Sapienza University of Rome,

Rome, Italy

e-mail: antonino.raffone@uniroma1.it

\section{N. Srinivasan}

Centre of Behavioural and Cognitive Sciences,

University of Allahabad, Allahabad, India

A. Raffone $\cdot$ N. Srinivasan

Perceptual Dynamics Laboratory, Brain Science Institute RIKEN, Tokyo, Japan mental and related somatic events are affected by engaging a specific attentional set. Many recent behavioral, electroencephalographic and neuroimaging studies have revealed the importance of investigating states and traits related to meditation in order to achieve an increased understanding of cognitive and affective neuroplasticity, attention and selfawareness, as well as for their possible clinical implications (Cahn and Polich 2006; Lutz et al. 2008a).

The regulation of attention is a central feature of different meditation methods (Davidson and Goleman 1977), and meditation practices can be usefully classified into two main styles-focused attention (FA) and open monitoring $(\mathrm{OM})$-depending on how the attentional processes are directed (Cahn and Polich 2006; Lutz et al. 2008a). In the FA ('concentrative') style, attention is focused on a given object in a sustained manner. The second style, OM ('mindfulness-based') meditation, involves the non-reactive monitoring of the content of ongoing experience, primarily as a means to become reflectively aware of the nature of emotional and cognitive patterns. Mindfulness refers to an alert and open mode of perceiving and monitoring all mental content from moment to moment, including perceptions, sensations, cognitions and affects (Kabat-Zinn 2003).

\section{Focused attention meditation}

Apart from sustaining the attentional focus on an intended object, FA meditation also implies the regulative skills of monitoring the focus of attention, detecting distraction, disengaging attention from the source of distraction, and (re)directing and refocusing on the object (Lutz et al. 2008a). FA meditation techniques involve observing the experiential field by allowing thoughts and sensations to 
arise and pass without clinging to them, maintaining attention on an object or bringing it back to the specific object of concentrative (or focused) awareness, in order to develop an internal 'witnessing observer' (Cahn and Polich 2006). The attentional and monitoring functions of FA meditation have been related to dissociable systems in the brain involved in conflict monitoring as well as general selective and sustained attention (Corbetta and Shulman 2002; Lutz et al. 2008a; Weissman et al. 2006).

It has been observed that the practice of FA meditation leads to reduced effort in sustaining the focus on an intended object. Expertise in FA meditation leads to a greater monitoring ability to detect arising distractions or mind wanderings, thus implying reduced cognitive efforts during practice (Lutz et al. 2008a). In FA meditation practice, high attentional stability and vividness are achieved in a mental state of concentrated calm or serene attention, denoted by the word Samatha (with the literary meaning of quiescence) in the Buddhist contemplative tradition (Wallace 1999). Wallace (1999) observes that the development of attentional stability may be likened to mounting a telescope on a firm platform, while the development of attentional vividness is like polishing the lenses and bringing the telescope into clear focus.

Transcendental meditation (TM) can be broadly included in the FA meditation category, as its practice centers on the repetition of a mantra. However, TM primarily emphasizes the absence of an effort to maintain the concentration and the development of a witnessing, thoughtfree 'transcendental awareness' or 'pure consciousness', which is characterized by the absence of any concentration or effort unfolding experiences of "unboundedness" and "loss of time, space and body sense" (Arenander and Travis 2004; see below for more explanations). These states are associated with profound physical relaxation, marked by spontaneous breath quiescence and global, high amplitude, slow frequency (alpha) EEG patterns which are general highly coherent across frontal leads (Arenander and Travis 2004; Travis and Pearson 1999; Travis and Wallace 1999). A similar reference to a pure or intuitive awareness can be found in Buddhism, as reflecting a "nonself' mental state, which is, however, associated with a mindfulness-based insight meditation (e.g., Sumedho 2004).

\section{Open monitoring meditation}

Open monitoring (OM) meditation does not involve an explicit attentional focus and therefore does not seem to be associated with brain areas implicated in sustained or focused attention. Instead, it involves brain regions implicated in vigilance, monitoring and disengagement of attention from sources of distraction from the ongoing stream of experience (Lutz et al. 2008a). OM practices are based on an attentive set that is characterized by an open presence and a non-judgmental awareness of sensory, cognitive and affective fields of experience in the present moment and involves a higher-order (meta-) awareness of ongoing mental processes (Cahn and Polich 2006). The cultivation of this 'reflexive' awareness in OM meditation is associated with a more vivid conscious access to the rich features of each experience and enhanced metacognitive and self-regulatory skills (Lutz et al. 2008a).

As introduced above, OM meditation is also referred to as mindfulness meditation (Cahn and Polich 2006). The notion of mindfulness, derived from Buddhist texts and always more emphasized in cognitive and clinical psychological contexts (e.g., Cahn and Polich 2006; KabatZinn 2003; Sumedho 2004), might provide a unifying construct for endogenous attention, monitoring and executive control functions (Raffone and Srinivasan 2009). It describes a virtue to be cultivated by meditation and practice in everyday life.

Unlike FA meditation, OM meditation does not entail attentional biases resulting in selection and de-selection processes. Therefore, in OM meditation, cognitive monitoring is reflected in an open-field capacity to detect arising sensory, feeling and thought events within an unrestricted 'background' of awareness, without a 'grasping' of these events in an explicitly selected foreground or focus. In the transition from a FA to an OM meditative state, the object as the primary focus is gradually replaced by an 'effortless' sustaining of an open background of awareness without an explicit attentional selection (Lutz et al. 2008a). Behavioral studies have shown a more distributed attentional focus (Valentine and Sweet 1999), enhanced conflict monitoring (Tang et al. 2007) and reduced attentional blink or more efficient resource allocation to serially presented targets (Slagter et al. 2007) in OM meditators.

In contemplative practice, as in the Buddhist tradition, attentional stability and vividness (acuity), as developed in FA meditation, are regarded as necessary for deep and reliable introspection to take place, as in the practice of Vipassana (insight) OM meditation. As indicated by Wallace (1999), Tsongkhapa, an eminent Tibetan Buddhist contemplative and philosopher, highlighted the importance of attentional stability and vividness for the cultivation of contemplative insight, by referring to an oil lamp which is both radiant and unflickering and which with its light allows shapes on a tapestry to be observed in detail and vividly at night. When the light is dim or the wind induces flickering, the perception of shapes is lost. In the Buddhist contemplative tradition, introspection, as performed in $\mathrm{OM}$ insight meditation, is regarded as a form of metacognition. 
States and traits in neuroscientific studies of meditation

Studies on the neuroscience of meditation can be broadly classified into two types: one type focuses on the nature of changes in the brain activity during the practice of meditation (meditation state) and the other type focuses on the cognitive and neural changes due to the long-term meditation practice (meditation trait). The joint neuroscientific investigation of meditation states and traits can take place in appropriately designed studies. For example, in the EEG study by Lutz et al. (2004), high-amplitude gamma-band oscillations and phase-synchrony were observed in longterm practitioners during meditation. These EEG patterns differed from those of controls, in particular over lateral frontoparietal electrodes. Furthermore, as a trait effect, the ratio of gamma-band activity $(25-42 \mathrm{~Hz})$ to slow oscillatory activity $(4-13 \mathrm{~Hz})$ was initially higher in the resting baseline before meditation for the long-term practitioners than the controls over medial frontoparietal electrodes. This difference increased sharply as a meditation state effect over most of the scalp electrodes and remained higher than the initial baseline in the post-meditation baseline.

A large number of studies have investigated the behavioral, cognitive and neural changes due to the longterm practice of meditation (e.g., Brefczynski-Lewis et al. 2007; Carter et al. 2005; Lutz et al. 2004; Slagter et al. 2007; Srinivasan and Baijal 2007). For example, Srinivasan and Baijal (2007) reported the changes in automatic preattentive processing due to concentration meditation. Frequent and infrequent auditory tones were presented to the meditators (while exiting meditation) and control group (during relaxation). The results showed increase in MMN (mismatch negativity) amplitudes immediately after and before meditation indicating enhanced sensitivity of the perceptual system of the meditators even before attention could be allocated for a cognitive task. Changes in perceptual processes have been shown with a study on Buddhist meditators in which expert meditators (Tibetan Buddhist monks) could sustain the motion-induced blindness for a long period (Carter et al. 2005), as a FA meditation state effect. The study also reported longer durations with a binocular rivalry task indicating possible changes in the brain mechanisms responsible for perceptual awareness, as a state effect of FA meditation based on (trait) meditation expertise (Carter et al. 2005). Other studies have found trait-related structural changes in the brain of long-term meditation practitioners by structural MRI (e.g., Lazar et al. 2005; Pagnoni and Cekic 2007).

Several studies have reported changes in attentional processes due to meditation practice. Meditators show decreased attentional blink, improved ability to control perceptual rivalry, reduced distractibility and more sustained attention (for a review see Lutz et al. 2008a). A functional magnetic resonance imaging (fMRI) study with the practitioners of FA meditation showed lower activations to regions related to distraction and task unrelated thoughts compared to the controls during a task that required the observers to ignore auditory stimuli (Brefczynski-Lewis et al. 2007). Practice of FA meditation resulted in enhanced ability to focus attention thereby reducing the effects of irrelevant stimuli. During FA meditation compared to rest, there was activation in multiple regions associated with monitoring and engagement of attention such as dorsolateral prefrontal cortex, visual cortex, superior frontal and intraparietal sulci. Interestingly, the strength of activity in these regions showed an inverted $u$-shaped curve with meditators with moderate experience showing stronger activation but very experienced meditators showing less activation. The $u$-shaped pattern of brain activity indicates that with very extensive mental training due to the practice of FA meditation, minimal effort might be necessary to sustain the focus of attention.

Another study with Vipassana (OM) meditation has shown a reduced attentional blink $(\mathrm{AB})$ a deficit in identifying the second of two targets (T1 and T2) presented in close succession, indicating efficient distribution of the limited attentional resources (Slagter et al. 2007). The Vipassana meditators possibly gained better control over the allocation of attention by reducing the resources devoted to the first target (T1) processing (as exhibited by reduced T1-related P3b amplitude) such that the subsequent target was more often detected (or reduced $\mathrm{AB}$ ). In a related study (Slagter et al. 2009), EEG spectral analyses showed that intensive mental training in the form of Vipassana meditation was associated with decreased cross-trial variability in the phase of oscillatory theta activity after successfully detected $\mathrm{T} 2 \mathrm{~s}$, in particular, for those individuals who showed the greatest reduction in brain resource allocation to T1. This finding implicates theta phase locking in conscious target perception and suggests that after meditation-based mental training the cognitive system is more rapidly available to process new target information. In another investigation, Lutz et al. (2009) found a reduced variability in attentional processing of target tones after intensive FA/OM meditation training, as showed by both enhanced theta-band phase consistency of oscillatory neural responses over anterior brain areas and reduced reaction time variability. Moreover, those participants who showed the greatest increase in neural response consistency showed the largest decrease in behavioral response variability.

The research reports on meditation in this issue (Bærentsen et al. 2010; Baijal and Srinivasan 2010; Cahn et al. 2010; Travis et al. 2010) investigate the neural 
changes as the practitioners meditate in the laboratory (Tagini and Raffone's article in this issue presents a related theoretical development). These studies employ both FA and OM meditation types. Part of the problem in integrating the results is that slightly different paradigms are used even with the use of a single methodology like EEG. Another problem is relating the findings from EEG and functional fMRI studies. The EEG studies look at spectral values in different frequency ranges or uses measures of coherence between brain activities recorded by different channels while the fMRI studies focus on the activations in different brain areas.

In spite of the differences in the EEG paradigms and types of meditation, there are some consistent findings across studies. For example, Baijal and Srinivasan (2010) recorded EEG during FA meditation and found increased theta activity in the frontal areas during the middle portion of the meditation period. With a completely different $\mathrm{OM}$ meditation (Vipassana), Cahn et al. (2010) also found increase in theta activity in the frontal areas. The studies also indicate differences in the rhythmic brain activity. For example, Baijal and Srinivasan (2010) found a decrease in theta activity in the parietal areas during the middle portion of the meditation period. Cahn et al. (2010) found an increase in gamma activity in the parieto-occipital areas. TM is classified somewhat as a FA type as discussed earlier given the use of mantra during meditation, and Travis et al. (2010) found higher alpha power and higher coherence in the frontal areas in the alpha and beta2 ranges. They also found lower power in betal and gamma ranges in the frontal areas.

Travis et al. (2010) found that cortical midline circuits activated during TM practice overlap those of the default mode network (Raichle et al. 2001), which has been defined as a fundamental or "intrinsic property of the brain" supporting the extrinsic modes of cognitive processing (Fox and Raichle 2007). Travis et al. concluded that since activation in these default mode brain areas was higher during TM compared to eyes-closed rest, the experience of contentless thought with continued selfawareness during TM practice must be different from autobiographical or mind-wandering thoughts. This conclusion might nicely converge with the theoretical synthesis and neurocognitive hypothesis presented by Tagini and Raffone (2010), though further neuroimaging investigations are needed.

The fMRI investigation by Bærentsen et al. (2010) elucidates fundamental aspects of meditation practice beyond specific styles, with a view of meditation as a practice to still the fluctuations of the mind, with reference to Patanjali's classical analysis of yoga. Baerentsen et al.'s study reveals distributed deactivation and activation patterns in the brain, in both cortical and subcortical regions, characterizing switching into the meditation state and sustaining it.

\section{Neuroscience of meditation and consciousness}

Apart from attentional sets, other neurocognitive processes underlying different aspects of consciousness and selfawareness are also implicated in meditation. Mindfulness and open monitoring in meditation are linked to a momentary (first person) self-awareness rather than to a narrative (third person) self-awareness (see Tagini and Raffone's theoretical article in this issue). A recent neuroimaging study was designed to characterize the neural bases of such two forms of self-awareness, i.e., narrative self-reference and momentary self-reference (Farb et al. 2007). Farb et al. investigated monitoring of enduring traits ('narrative' focus, NF) or momentary experience ('experiential' focus, EF) in both novice participants and participants having attended an 8-week course in mindfulness meditation. In novices, EF resulted in focal reductions in the activation of self-referential cortical areas associated with NF. In trained participants, EF yielded more pronounced reductions in medial prefrontal cortex activation. Following mindfulness training, EF resulted in an activation pattern supporting a more objective and self-detached analysis of sensory events, rather than their subjective or affective self-referential evaluation. As suggested by Farb et al.'s (2007) findings, awareness of subjective or phenomenal aspects of experience in the present moment involves neuronal populations with responses marking transient body states, in particular, right lateralized exteroceptive somatic and interoceptive insular cortices (Craig 2004; Critchley et al. 2004; Damasio 1999). Somatic marker or momentary self-awareness areas have also been implied in OM or mindfulness-based meditation (Lutz et al. 2008a).

Baars $(1998,2002)$ hypothesized that conscious events involve self-systems in the brain and in particular a "narrative interpreter' in the left hemisphere (with involvement of prefrontal cortex). Metaphorically, this interpreter would operate as a stage director in a theater. Baars particularly referred to Gazzaniga's (1985) findings with split-brain patients and argued that the two hemispheres might each possess an 'observer' of the respective conscious flow of visual information. To address the issue that conscious perception may entail a dialog between specific self-related prefrontal regions ('stage director' or executive interpreter) and sensory cortex (Baars et al. 2003), brain activity patterns produced by a demanding sensory categorization task were compared to those engaged during self-reflective introspection, using similar sensory stimuli (Goldberg et al. 2006). The results showed a complete segregation between 
the two brain activity patterns thus challenging Baars' hypothesis of an involvement of self-related observer-like prefrontal regions in perceptual awareness. Moreover, areas characterized by enhanced activity during introspection exhibited a robust inhibition during the demanding perceptual task, thus suggesting that self-related brain activities are not necessarily implied in perceptual awareness, and that they can be suppressed. Interestingly, Goldberg et al. (2006) relate their findings to the Zen Buddhist view on selflessness: “...the picture that emerges from the present results is that, during intense perceptual engagement, all neuronal resources are focused on sensory cortex, and the distracting self-related cortex is inactive. Thus, the term "losing yourself" receives here a clear neuronal correlate. This theme has a tantalizing echoing in Eastern philosophies such as Zen teachings, which emphasize the need to enter into a "mindless," selfless mental state to achieve a true sense of reality..." (p. 337).

Since when we view a complex visual scene we experience a richness of content that seems to go beyond what we can report, Block proposed a distinct state of phenomenal consciousness prior to global conscious access (see Block 1995, 2007, for the distinction between phenomenal and access consciousness). Access conscious contents are instead characterized in terms of information that is available to the brain's 'consumer' systems (e.g., decision making) and to the rational control of action. Raffone and Srinivasan (2009) and Raffone et al. (2009) recently suggested that phenomenal consciousness is implied in the moment to moment open 'background' awareness in open monitoring meditation (see also above), based on a distributed rather than focused attention (Srinivasan et al. 2009).

Siegel (2007) has suggested a distinction between three different forms of awareness with reference to mindfulness-based Buddhist meditation (Cahn and Polich 2006; Lutz et al. 2008a). These are a receptive or mindful awareness, with openness to whatever "comes to mind in the moment", which has been shown to create a state of flexibility in self-regulation enabling an individual to profoundly shift out of habitual ways of adapting and reacting (e.g., Kabat-Zinn 2003); a self-observational awareness, accompanied by reflective self-observation, including the metacognitive investigation of one's mental processes; the integration of this self-reflective state with receptivity is characterized by curiosity, openness, acceptance and love (COAL); a reflexive awareness, implying a more immediate capacity of the mind to know itself, without effort and words, leading to an understanding of the nature of 'awareness of awareness'.

Raffone and Srinivasan (2009) recently suggested that a metacognitive consciousness, related to the self-observational aspects of mindfulness, may refer to being aware of either an object or to the subjective experience of cognition of an object. This metacognitive consciousness is regarded as 'transversal' to any form of awareness based on the subject-object cognitive duality, whether it refers to an external or internal object per se (first-order consciousness), or to the subjective or phenomenal experience of such an object (second-order consciousness). Such non-referential awareness would thus correspond to a third-order consciousness, going beyond the cognitive subject-object duality, as the 'awareness of being aware' (Arenander and Travis 2004; Zeki 2003). In our view, this transcendent awareness may only be developed through meditationbased intuition and can thus also be characterized as an intuitive awareness (Sumedho 2004), or as the reflexive awareness facet of mindfulness (Siegel 2007).

The neural correlates of different aspects of phenomenal, access and metacognitive consciousness as characterized in the adaptive workspace hypothesis might be clarified in the context of FA and especially OM meditation settings, with highly trained meditators capable of switching between different consciousness modes, with attention to external sensory fields or internal (thought- and feeling-related) fields of experience, by using the neurophenomenology approach (Lutz and Thompson 2003; Varela 1996). In the neurophenomenology approach, quantitative measures of neural activity are combined with first-person data about the subject's inner experience. Participants' reports can thus be useful in identifying variability in brain activity from moment to moment; this unique information might guide the detection and interpretation of neural processes correlated to different aspects of conscious experience. Novel techniques for EEG analysis might be fruitfully applied in that framework (Fingelkurts and Fingelkurts 2006; Thompson and Varela 2001).

In further studies it may be insightful to compare brain activity patterns in OM meditation conditions with differential awareness of fields of experience, such as body sensory field, external sensory fields and 'internal' thoughts and feelings. Moreover, it appears useful to design an experiment comparing brain activity patterns in FA meditation with focus on breathing-related sensations and on an external visual point. Large-scale computational models with biological and cognitive constraints related to experimental evidence about attention and conscious access processes (e.g., Dehaene et al. 2003) would also contribute to shed light on the neural mechanisms implied in FA and OM meditation.

Finally, an increased scientific understanding of compassion (loving kindness) meditation and related brain circuitries appears important in light of relevant relationships with empathy and theory of mind (Lutz et al. 2008b) and clinical implications of compassion-based practice (Gilbert and Procter 2006). It also appears relevant to 
integrate such an understanding with knowledge about the mechanisms of focused attention and monitoring investigated, for example, in TM, Samatha and Vipassana meditation forms.

\section{References}

Arenander A, Travis FT (2004) Brain patterns of self-awareness. In: Beitman B, Nair J (eds) Self-awareness deficits. W.W. Norton, New York

Baars B (1998) Metaphors of consciousness and attention in the brain. Trends Neurosci 21:58-62

Baars BJ (2002) The conscious access hypothesis: origins and recent evidence. Trends Cogn Sci 6:47-52

Baars BJ, Ramsoy TZ, Laureys S (2003) Brain, conscious experience and the observing self. Trends Neurosci 26:671-675

Bærentsen KB et al (2010) An investigation of brain processes supporting meditation. Cogn Process (this issue)

Baijal S, Srinivasan N (2010) Theta activity and meditative states: spectral changes during concentrative meditation. Cogn Process (this issue)

Block N (1995) On a confusion about a function of consciousness. Behav Brain Sci 18:227-287

Block N (2007) Consciousness, accessibility, and the mesh between psychology and neuroscience. Behav Brain Sci 30:481-548

Brefczynski-Lewis JA et al (2007) Neural correlates of attentional expertise in long-term meditation practitioners. Proc Natl Acad Sci USA 104:11483-11488

Cahn BR, Polich J (2006) Meditation states and traits: EEG, ERP, and neuroimaging studies. Psychol Bull 132:180-211

Cahn BR, Delorme A, Polich J (2010) Occipital gamma activation during Vipassana meditation. Cogn Process (this issue)

Carter OL et al (2005) Meditation alters perceptual rivalry in Tibetan Buddhist monks. Curr Biol 15:R412-R413

Corbetta M, Shulman GL (2002) Control of goal-directed and stimulus-driven attention in the brain. Nat Rev Neurosci 3:201-215

Craig AD (2004) Human feelings: why are some more aware than others? Trends Cogn Sci 8:239-241

Critchley HD, Wiens S, Rotshtein P et al (2004) Neural systems supporting interoceptive awareness. Nat Neurosci 7:189-195

Damasio A (1999) The feeling of what happens: body and emotion in the making of consciousness. Harcourt, Brace, New York

Davidson RJ, Goleman DJ (1977) The role of attention in meditation and hypnosis: a psychobiological perspective on transformations of consciousness. Int J Clin Exp Hypn 25:291-308

Dehaene S, Sergent C, Changeux J-P (2003) A neuronal network model linking subjective reports and objective physiological data during conscious perception. Proc Natl Acad Sci USA 100:8520-8525

Farb NAS, Segal ZV, Mayberg H et al (2007) Attending to the present: meditation reveals distinct neural modes of selfreference. Soc Cogn Affect Neurosci 2:313-322

Fingelkurts AA, Fingelkurts AA (2006) Timing in cognition and EEG brain dynamics: discreteness versus continuity. Cogn Process 7:135-162

Fox M, Raichle M (2007) Spontaneous fluctuations in brain activity observed with functional Magnetic Resonance Imaging. Nat Rev Neurosci 8:700-711

Gazzaniga MS (1985) The social brain. Basic Books, New York

Gilbert P, Procter S (2006) Compassionate mind training for people with high shame and self-criticism: overview and pilot study of a group therapy approach. Clin Psychol Psychother 13:353-379
Goldberg II, Harel M, Malach R (2006) When the brain loses its self: prefrontal inactivation during sensorimotor processing. Neuron 50:329-339

Kabat-Zinn J (2003) Mindfulness-based interventions in context: past, present, and future. Clin Psychol Sci Pract 10:144-156

Lazar SW, Kerr CE, Wasserman RH et al (2005) Meditation experience is associated with increased cortical thickness. Neuroreport 16:1893-1897

Lutz A, Thompson E (2003) Neurophenomenology: integrating subjective experience and brain dynamics in the neuroscience of consciousness. J Conscious Stud 10:31-52

Lutz A, Greischar L, Rawlings NB, Ricard M, Davidson RJ (2004) Long-term meditators self-induce high-amplitude synchrony during mental practice. Proc Natl Acad Sci USA 101:1636916373

Lutz A, Slagter HA, Dunne JD, Davidson RJ (2008a) Attention regulation and monitoring in meditation. Trends Cogn Sci 12:163-169

Lutz A, Brefczynski-Lewis J, Johnstone T, Davidson RJ (2008b) Regulation of the neural circuitry of emotion by compassion meditation: effects of meditative expertise. PLoS ONE 3:e1897

Lutz A, Slagter HA, Rawling BN, Francis DA, Greischar LL, Davidson RJ (2009) Mental training enhances stability of attention by reducing cortical noise. J Neurosci 29:13418-13427

Pagnoni G, Cekic M (2007) Age effects on gray matter volume and attentional performance in Zen meditation. Neurobiol Aging 28:1623-1627

Raffone A, Srinivasan N (2009) An adaptive workspace hypothesis about the neural correlates of consciousness: insights from neuroscience and meditation studies. In: Srinivasan N (ed) Progress in brain research: attention, vol 176. Elsevier, Amsterdam, pp 161-180

Raffone A, Tagini A, Srinivasan N (2009) Mindfulness and the cognitive neuroscience of attention and awareness. Zygon (in press)

Raichle ME, MacLeod AM, Snyder AZ, Powers WJ, Gusnard DA, Shulman GL (2001) A default mode of brain function. In: Proceedings of the National Academy of Sciences, vol 98. USA, pp 676-682

Siegel DJ (2007) The mindful brain. Norton, New York

Slagter HA et al (2007) Mental training affects distribution of limited brain resources. PLoS Biol 5:e138

Slagter HA, Lutz A, Greischar LL, Nieuwenhuis S, Davidson RJ (2009) Theta phase synchrony and conscious target perception: impact of intensive mental training. J Cogn Neurosci 21:1536-1549

Srinivasan N, Baijal S (2007) Concentrative meditation enhances preattentive processing: a mismatch negativity study. Neuroreport 18:1709-1712

Srinivasan N, Srivastava P, Lohani M, Baijal S (2009) Focused and distributed attention. In: Srinivasan N (ed) Progress in brain research: attention, vol 176. Elsevier, Amsterdam

Sumedho A (2004) Intuitive awareness. Amaravati Buddhist Monastery, Hemel Hempstead

Tagini A, Raffone A (2010) The 'I' and the 'Me' in self-referential awareness: a neurocognitive hypothesis. Cogn Process (this issue)

Tang YY et al (2007) Short term meditation training improves attention and self regulation. In: Proceedings of the National Academy of Sciences, vol 104. USA, pp 17152-17156

Thompson E, Varela FJ (2001) Radical embodiment: neural dynamics and consciousness. Trends Cogn Sci 5:418-425

Travis F, Pearson C (1999) Pure consciousness: distinct phenomenological and physiological correlates of "consciousness itself". Int J Neurosci 100:77-89

Travis F, Wallace RK (1999) Autonomic and EEG patterns during eyes-closed rest and transcendental meditation (TM) practice: 
the basis for a neural model of TM practice. Conscious Cogn $8: 302-318$

Travis $\mathrm{F}$ et al (2010) A self-referential default brain state: patterns of coherence, power, and eLORETA sources during eyes-closed rest and Transcendental Meditation practice. Cogn Process (this issue)

Valentine ER, Sweet PLG (1999) Meditation and attention: a comparison of the effects of concentrative and mindfulness meditation on sustained attention. Ment Health Relig Cult 2:59-70
Varela F (1996) Neurophenomenology: a methodological remedy to the hard problem. J Conscious Stud 3:330-350

Wallace A (1999) The Buddhist tradition of Samatha: methods for refining and examining consciousness. J Conscious Stud 6:175-187

Weissman DH et al (2006) The neural bases of momentary lapses in attention. Nat Neurosci 9:971-978

Zeki S (2003) The disunity of consciousness. Trends Cogn Sci 7:214-221 